\title{
Germanica
}

\section{Vienne selon Hofmannsthal : un adieu, entre critique et nostalgie}

Wien im Werke Hofmannsthals, ein kritischer und sehnsüchtiger Abschied

\section{Audrey Giboux}

\section{(2) OpenEdition}

\section{Journals}

Édition électronique

URL : http://journals.openedition.org/germanica/554

DOI : $10.4000 /$ germanica.554

ISSN : 2107-0784

Éditeur

Université de Lille

Édition imprimée

Date de publication : 1 décembre 2008

Pagination : 53-63

ISBN : 978-2-913857-22-6

ISSN : 0984-2632

\section{Référence électronique}

Audrey Giboux « Vienne selon Hofmannsthal : un adieu, entre critique et nostalgie », Germanica [En ligne], 43 | 2008, mis en ligne le 01 décembre 2010, consulté le 06 octobre 2020. URL : http:// journals.openedition.org/germanica/554; DOI : https://doi.org/10.4000/germanica.554

Ce document a été généré automatiquement le 6 octobre 2020 .

(ㄷ) Tous droits réservés 


\title{
Vienne selon Hofmannsthal : un adieu, entre critique et nostalgie
}

Wien im Werke Hofmannsthals, ein kritischer und sehnsüchtiger Abschied

\author{
Audrey Giboux
}

\section{Hofmannsthal le Viennois}

1 Né à Vienne en 1874, Hofmannsthal grandit Salesianergasse, et est scolarisé au prestigieux Akademisches Gymnasium. Dès 1890, il entre dans le cercle de Bahr, «das Junge Wien ", bientôt stigmatisé par Kraus ${ }^{1}$ : les clichés le représentant au café Griensteidl sur la Michaelerplatz, entouré de Schnitzler, Andrian, Beer-Hofmann, Salten, sont célèbres. Après son mariage en 1901, il s'installe à la campagne à Rodaun, tout en conservant son appartement de la Stallburggasse. Il se considère toujours comme un citoyen viennois ; en témoignent ses textes de guerre, qui manifestent sa solidarité avec les conditions de vie précaires des Viennois (RAII, p. $347^{2}$ ) ; après avoir servi en Croatie comme officier, il est nommé à un poste bureaucratique au ministère de la guerre ${ }^{3}$.

2 S'il n'a pas écrit de texte canonique sur sa ville natale, il l'évoque pourtant souvent. Considéré comme l'un des plus éminents représentants d'une « Modernité viennoise » qu'il ne revendique nullement, il (se) représente sa ville d'une manière ambiguë. Estelle pour lui la capitale de « l'enterrement au berceau de la modernité $»^{4}, \mathrm{du}$ " vide des valeurs » et de la " joyeuse apocalypse $»^{5}$ ? Parvient-il à formuler une identité viennoise qui soit irréductible à la germanité?

\section{Images et stéréotypes de Vienne}

3 Aimant rappeler le statut historique de Vienne, capitale du Saint Empire porteur d'un idéal universaliste (RAII, p.378), Hofmannsthal voit en Marie-Thérèse sa figure tutélaire, maternelle (RAII, p. 443). Il vante le creuset des nationalités qu'a été la Vienne d'avant-guerre: porta orientis, elle a, avec Hammer-Purgstall, suscité la vogue 
orientaliste, et est restée le havre des études slaves; elle est, comme Paris, une métropole supranationale (RAII,p.474). Douloureusement affecté par la chute de l'Empire, il affirme que l'Autriche doit devenir le ferment de la reconstruction européenne (RAII,p. 454). Son désarroi trouve un exutoire dans la tentative de cerner l'originalité des visages de Vienne - entreprise périlleuse au vu de la difficulté à définir l'identité autrichienne au sein de la germanité; il assimile tantôt la nationalité autrichienne à l'identité allemande (RAI,p. $412 ;$ p. 494), tantôt revendique la singularité de son pays, son goût pour la simplicité, la naïveté (RAI, p.139), en s'appuyant sur l'opposition entre artistes allemands et autrichiens (RAII, p. 87).

4 Il recourt parfois à une imagerie pittoresque, où le fiacre est défini comme un «gondolier viennois » (RAIII, p. 323), et s'attache à saisir la mentalité viennoise en reconduisant quelques clichés: le Viennois typique est supposé dépourvu de pédanterie, assez impertinent pour «faire le polichinelle» (RAII, p.535), plus cérémonieux que poli (RAIII, p. 525), et de renvoyer au distinguo de Schiller selon lequel les Allemands sont touchés sur le plan moral, quand les Autrichiens le sont par l'esthétique (RAIII, p. 527). Non sans autodérision, Hofmannsthal imagine ce scénario, où le Prussien, selon lui, ne perdrait jamais son assurance même dans l'erreur :

Un Viennois prononce le nom d'un peintre étranger comme il croit l'avoir entendu dire par les érudits; il arrive parmi les compatriotes du peintre, améliore sa prononciation d'après la leur, rentre parmi les Viennois et abandonne sa prononciation devenue correcte [...]. Tout ceci à demi par politesse, à demi par refus de vaincre une résistance ${ }^{6}$.

Plus sérieusement, il fait de l'Anatol de Schnitzler, «dandy sentimental », un archétype de l'âme viennoise populaire - une figure esthétisée, loin de la "viennoiserie» (Wienerthum) colportée par les journalistes et les chanteurs populaires :

[L]e style de ses pensées a quelque chose qui rappelle légèrement les Français, mais sans la tristesse amère et grimaçante des modernes [...] : tout fonctionne sur trois temps, comme une valse solennelle, avec une mélancolie souriante [...]. Car cette âme viennoise-ci respire entre les lignes : celle, timide et sensible à l'excès, rêvée, du Pauvre musicien et des aquarelles d'Alt. Non pas l'autre, l'âme brutale [...]. Ni non plus l'âme laïque et gracieuse [...]

6 La spécificité des avatars viennois n'est pas qu'affaire de psychologie : elle est fondée sur un idiome. La langue viennoise, distinguée de l'allemand (langue de l'individuel, audelà de toute communication, au contraire du français, langue de la communauté, de la compréhension), est marquée par une prononciation particulière, des particularismes grammaticaux (RAIII, p. 250), et mêle la langue des diplomates, militaires, bourgeois, aristocrates, se nuançant en fonction des quartiers de Vienne, déterminant une "atmosphère", un cosmopolitisme linguistique: "Au sein des possessions de notre langue se cache tout un fouillis de mots étrangers, mais ce sont nos mots étrangers, ils sont, chez nous, comme chez eux depuis des siècles et à tel point devenus nôtres qu'ils ont perdu leur citoyenneté dans leur propre patrie » (RAII, p. 364). Louant la richesse de ces sociolectes et non leur pouvoir discriminant, Hofmannsthal vante la faculté du public viennois à saisir toutes les subtilités de la langue, du mime et de la musique, qui a contribué à la popularité mondiale de l'opérette viennoise (RAII, p. 273). 


\section{L'aura viennoise...}

7 Vienne bénéficie aux yeux de Hofmannsthal d'un sentiment d'élection. En témoigne l'attention aiguë qu'il porte à la psychanalyse, dont elle semblait prédestinée à accueillir la naissance :

[Vienne] est la porta orientis aussi pour cet Orient secret, le royaume de l'inconscient. Les interprétations et hypothèses du $\mathrm{D}^{\mathrm{r}}$ Freud sont les excursions de l'esprit du temps conscient aux rives de ce royaume. [...] Rien ne dépend aussi étroitement du social et de la sociabilité que la psychologie, qui n'est rien d'autre que l'utilisation systématique des dons sociaux. Le sentiment pour autrui, l'attention portée à ses plus tendres émotions et une certaine capacité d'identification, d'où émerge ce qui ne s'apprend pas: le tact. Rien ne me semble plus naturel que de [constater] que [...] tant de contact se soit instauré entre un foyer viennois de la recherche et les écoles d'Europe de l'Ouest, comme avant tout avec celle de Paris $[. . .]^{8}$.

Le tact viennois est érigé en symptôme d'un genius loci favorable aux théories freudiennes, en partie fondées sur une relecture des mythes grecs. La métaphore de l'inconscient en Orient mystérieux de la pensée initie une mythification de Vienne en cité représentative de son époque, mais aussi en véritable topique du psychisme, incarnation d'un inconscient de l'Occident.

9 Hofmannsthal s'intéresse en outre à l'actualité culturelle et à tous les aspects de la création à Vienne, même à son artisanat (RAII, p. 57), sa porcelaine (RAI, p. 185). La peinture l'occupe particulièrement: il fait l'éloge des collections du Belvédère(RAII, p. 342), rend compte des expositions viennoises (RAI, p. 546). Parlant, curieusement, très peu de la Sécession (RAII, p. 318), il procède à un état des lieux de la peinture viennoise contemporaine (RAI, p.563), dont il juge les représentants avec peu d'indulgence :

La peinture viennoise a étonnamment peu de caractère [...]. Et pourtant ces gens et même cette ville sont si particuliers! [...] La peinture de genre viennoise [...] ne montre pas des gens, mais les figures fixes de la mauvaise comédie allemande; des personnages impossibles, vêtus de manière impossible, dans des situations factices impossibles, [...] avec un air gauche, comme des dilettantes qui [...] s'efforcent au prix de pénibles luxations intérieures et extérieures d'être naturels et compréhensibles. La seule image viennoise qui parvienne à incarner la particularité de notre vie est celle d'Engelhart, Les Joueurs de cartes. Par ailleurs le tableau n'est pas en soi d'une qualité extraordinaire; mais il montre la meilleure école pour saisir les gestes et l'âme des personnes qui nous entourent: celle des grands caricaturistes français [...]. Si M. Engelhart est en mesure de s'acclimater à l'atmosphère viennoise [...], l'Autriche possédera un grand et précieux artiste9 .

La scène musicale occupe pareillement Hofmannsthal : il rend hommage à Mahler à la tête du Wiener Oper (RAI, p. 645), rappelle le rôle joué par l'esprit de Haydn et de Mozart hantant la ville lors du séjour de Beethoven à Vienne, seul dans la foule tel «Philoctète sur son île » (RAII, p. 78). C'est par sa musique, solennelle mais aimable, que Vienne a " conquis le monde", en mêlant à la source germanique "un souffle slave, un éclat italien » (RAII, p. 13).

La poésie autrichienne ne s'est développée que secondairement, avec un ancrage paysan. Le particularisme autrichien n'est pas celui de la cour comme à Berlin, mais dans une filiation pastorale en réaction contre le centralisme libéral. L'austrianisme chez Hofmannsthal, qui se défend de tout provincialisme réactionnaire ou nationaliste, 
correspond explicitement à une quête du génie, de la mythologie nationale, dans un modèle d'intégration linguistique calqué sur celui de la France.

Aussi, l'affirmation "Vienne est une ville de théâtre ", de polémique, est devenue une réalité historique, par laquelle le peuple s'exprime socialement, spirituellement (RAII, p. 13). Hofmannsthal se préoccupe vivement de l'actualité théâtrale - à laquelle il prend part, rendant compte des collections théâtrales du fonds de la Nationalbibliothek (RAII, p. 325), des enjeux du répertoire viennois (RAII, p. 109), de la venue d'Eleonora Duse lors d'un festival (RAI, p. 469). Il rend hommage aux acteurs viennois (RAI, p. 506), évoquant les particularités de leur jeu, qui puise ses racines dans le mime (RAII, p. 279). Il prend part aux débats publics quant aux changements de direction du Burgtheater en 1918, dont il déplore le déclin après qu'il a, sur plus d'un siècle, fait exceptionnel dans un pays germanophone, vécu sous une «bonne étoile»; rappelant son lien historique avec la cour de la Maison impériale et sa parenté avec la Comédie-Française (RAII, p. 274), il loue sa capacité à mêler grande culture et sens du naturel et à accueillir un public plus varié que le Königliches Schauspielhaus de Berlin (RAII, p. 236). Évoquant l'heureuse mixité générique du théâtre viennois, la cohabitation de tous les arts en son sein, il vante le théâtre vivant qui en découle, sa "génialité » qu'il compare aux théâtres grec et celtique (RAII, p. 272). Cet éloge prolonge celui du théâtre populaire, défini comme un traditionalisme éclectique, dans une osmose entre la pièce, l'acteur et le public (RAII, p. 252), chez les grands dramaturges viennois : Grillparzer (RAII, p. 87), Raimund (RAII, p. 116). Schnitzler en revanche, qui ne met en scène que la bourgeoisie intellectuelle, n'est représentatif de l'esprit viennois qu'en ce qu'il fait du théâtre une forme symbolique qui met en abyme la comédie des rapports sociaux (RAII, p. 276).

Plus généralement, Hofmannsthal chante la splendeur de Vienne :

La ville merveilleuse, aux enchantements inépuisables, à l'atmosphère mystérieuse, douce, baignée de lumière! Et sous le ciel de printemps clair comme dans un rêve, ces palais baroques gris-noirs, aux grillages d'airain et aux moucharabys à fioritures, aux armoiries de pierre [...] ! Ces cours anciennes, emplies du clapotis des fraîches fontaines, avec leurs taches de soleil, leur lierre et leurs cupidons ! Et dans le faubourg, ces petites maisons jaunes de l'époque de l'Empereur François, [...] mélancoliques, inquiétantes, de petits-bourgeois! Et dans le déclin du jour, ces recoins et impasses fascinants où les passants perdent soudain leur matérialité, leur banalité et d'où il émane un charme indicible, d'un chiffon rouge accroché à une fenêtre sale ! Et plus tard dans la soirée, le crépuscule des rives de Vienne : sur le lit noir et vide du fleuve, le noir enchevêtrement des buissons et des arbres [...] sur un fond transparent, irréel, de vapeurs jaunes et grises, et, dominant tout cela, les trois coupoles sombres et harmonieuses de Saint-Charles-Borromée ! Et tout cela, tant de grandeur et d'attraits, tant d'aimable grâce, tant d'échappées nostalgiques, une beauté si dense et riche de sens, [...] suffisamment de matière pour exprimer la complexité des âmes d'une génération tout entière, faut-il que personne ne le voie, ne le sente ? ${ }^{10}$

Cette harmonie picturale entre l'architecture la plus raffinée et un lien sensible avec la nature décrit Vienne en cité onirique, entre le mystère baroque et la pastorale romantique. Hofmannsthal affirme d'ailleurs que l'atmosphère viennoise n'a autant d'affinité avec aucune autre époque qu'avec la pompe baroque (RAII, p. 283). Ayant comparé le festival Die Wiener Woche aux Dionysies d'Athènes (RAI, p. 475), il transpose à Vienne, dans un poème en prose, «Intermezzo », la vision d'une cité antique :

Charles-Borromée de nuit devant le pont Schwarzenberg. Comme, dans une fastueuse sérénité, elle domine le gouffre vert et bleu de Vienne, [...] ce chemin tracé par l'eau [...] vers quelque plage noire et euxinique, barbare et lointaine comme un rêve, où 
vit le passé dans une atmosphère somnambulique! Et, flottant contre la chair de l'église, les épaisses cimes des arbres et les broussailles emplies des désirs, rires et souffrances de l'amour : comme, en cet endroit [...], nous saisissent tout à la fois le passé et le présent, ce qui est lointain et héroïque, ce qui est proche et humble, comme tout ici est vivant, vrai, uni, unifié par le medium de la sensation!

Un peuplier d'un noir bleuté tient la lune attachée à sa pointe. Elle a quelque chose d'étrange, de grec. Cela dit: Alciphron, Alcinoos, Alcibiade. [...] Trois mots chevauchant les montagnes barbares, loin de la terre natale, mais hardis, heureux du départ, songeant à [...] une certaine ivresse et à une gloire raffinée, spirituelle... ${ }^{11}$

La mythification va jusqu'à constituer Vienne en objet poétique; Hofmannsthal prétend suggérer sa propre "philosophie de la métaphore » en imaginant un dialogue platonicien entre deux amis errant émerveillés dans Vienne, qui produirait « un poème, un hymne vibrant sans objet » :

[0]n devrait les laisser se promener dans le Volksgarten de Vienne [...]. L'air brille d'un éclat spécifique et apporte dans cet espace gigantesque un envoûtement intime et mystérieux. [...] Une légère brise tiède résonne dans les cimes et [...] lève un fugace parfum de jasmin, de lilas et d'acacia. Puis revient le silence. Mais à la fin, saisis par tant de beauté, ces jeunes gens doivent commencer à parler d'art, comme les colonnes de Memnon doivent chanter lorsque la lumière les rencontre. Et ils sont [...] si baignés de métaphores, si accoutumés à contempler leur âme par l'entremise d'étranges analogies, à régler le chemin de leur vie sur des formes apocalyptiques menaçantes, attirantes; ils disent au monde : «Tu es mon rêve, je peux [...] te transporter comme une toile peinte ", et au vent du printemps : « Tu es l'amour juvénile ", à une église ancienne : «Ton crépuscule est plein de nostalgie », et à une jeune fille, des paroles qui conviennent à la musique, aux cerisiers en fleurs, à une gorgée d'eau; les paroles sont pour eux des êtres vivants, et ils fuient devant les concepts, comme devant de grands chiens noirs ${ }^{12}$.

16 Cette Vienne de conte de fées, qui évoque Thèbes, après en avoir appelé à la peinture, suscite également la poésie, devenant elle-même le support synesthésique et syncrétique de la métaphore esthétique.

\section{... et sa démystification}

17 En la dépeignant atemporelle ou anachronique, Hofmannsthal ne cherche pas à idéaliser une Vienne de carte postale et sait porter sur elle un regard sans complaisance. Il avoue, dès sa jeunesse, sa lassitude quant à la vie viennoise : « La vie que nous vivons à Vienne est mauvaise. [...] Intellectuellement, nous vivons comme des cocottes qui ne se nourrissent que de salade française et de sorbet $»^{13}$. L'ancrage terrien dégénère parfois en une pesanteur traditionaliste ou philistine (RAII, p. 130). Malgré son riche passé, la vie artistique de la Vienne du début du $\mathrm{xx}^{\mathrm{e}}$ siècle ne satisfait pas pleinement Hofmannsthal, qui la compare à un « membre refroidi, qui n'est plus irrigué de sang »; la "malédiction » selon laquelle elle " ne serait pas un marché pour l'art » est dénoncée comme un slogan responsable de la frilosité du public face à l'art moderne, de son incapacité à identifier la fonction épiphanique de la peinture, depuis des décennies où «il tombe sur le sol viennois autant de la lumière du grand art européen que dans un sombre réduit à travers la fissure d'une porte » (RAI, p. 526-527).

Conscient de l'ambiance délétère de sa ville (RAIII, p. 392), Hofmannsthal développe, sur le mode de la déploration, un imaginaire de fin du monde, de la ruine paradisiaque (RAIII, p. 373). Les délices de Vienne apparaissent dans leur décadence, leur corruption. Assimilée au jardin des Hespérides - censé marquer la limite de l'Occident, ce qui 
renvoie à l'ambiance crépusculaire de la capitale autrichienne -, Vienne, telle un clown triste, une courtisane vieillissante parce que désertée par la poésie, en a gagné une majesté paradoxale; la conscience de la modernité est ambiguë, sous le signe de la perte :

Cette ville ingénue, douce, avec ses nombreuses coupoles et ses jardins nostalgiques, dont les sommets sont effleurés du souffle de la musique, [...] aux images de la mère de Dieu souriant entre les lampes multicolores, cette «Vienne Capoue » doit avoir notablement vieilli depuis qu'elle n'a pas eu de nouveau grand poète. Comme si, un moment, [...] personne n'avait été là pour cueillir à l'arbre les pommes d'or de jouvence et les offrir à la ville corruptrice, séduisante. [...] Ses traits ont perdu l'éclat de l'ingénuité, et sous ses cheveux emmêlés, blond cendré, elle n'a plus ce front innocent, ces yeux si jeunes, ce nez camus de Pierrette : un trait d'une sorte de souffrance a mûri, spiritualisé sa beauté; les ailes de son nez se sont affinées et frémissent silencieusement, et dans ses yeux il est parfois une hauteur de sphin $x^{14}$.

On songe à la représentation érotisée du labyrinthe vénitien dans le roman Andreas, dont le héros, un jeune Viennois, s'avoue une fascination trouble pour le théâtre vénitien, une attirance qu'il n'assumerait pas dans sa ville natale : Venise, ville vouée à périr, devient dans l'initiation du personnage l'autre de Vienne, la radicalisation de ses penchants, son double sulfureux, le lieu de la transgression et de la subversion des valeurs.

Hofmannsthal va jusqu'à fantasmer Vienne en ville morte, au-delà du cliché de la ruine romantique. Sa génération, comme née trop tard dans un monde trop vieux, porte la difficile mission de faire revenir l'art en ses murs :

Quelle génération d'artistes désespérée (exaspérée) nous sommes, nous qui nageons dans le fracas confus de la tempête de notre époque, « la couronne de l'art entre les dents ». Ceux qui viendront après nous seront plus grands que nous; mais nous sommes pourtant depuis ceux du Sturm und Drang les premiers artistes complets. [...] Nous sommes peut-être les derniers individus complets, dotés d'une âme, [...] ensuite peut-être surviendra une grande barbarie, un monde judéo-slave, sensuel. Penser Vienne comme une ville détruite : tous les murs écroulés, la chair intime de la ville mise à nu, les blessures recouvertes d'une végétation grimpante infinie, partout des frondaisons d'un vert lumineux, du silence, le clapotis de l'eau, toute vie enfuie [...] ! Et être les gardiens dans une des colonnes trajanes encore intactes devant Charles-Borromée, et déambuler dans les ruines avec des pensées qu'ici plus personne ne comprend ${ }^{15}$.

21 Il se représente en veilleur d'un temps d'obscurité, donnant de l'avenir une représentation apocalyptique, tributaire de certains clichés d'époque. Vienne, née de la barbarie, semble vouée à voir sa civilisation s'effondrer.

Hofmannsthal a situé relativement peu d'actions de ses pièces à Vienne, mais les exceptions accréditent la stylisation de cette ville en symbole d'une époque et d'une société disparues. À l'opéra, l'intrigue du Chevalier à la rose se déroule dans la Vienne de Marie-Thérèse, celle d'Arabella vers 1860, ce qui participe d'une " déterritorialisation » de Vienne dans le passé et montre, dans le premier cas, la nostalgie de l'auteur pour une époque de grandeur et de candeur révolues, marquée par le pastiche euphorique de la langue de l'époque, et, dans le second, sa conscience nouvelle des enjeux de la cohabitation des nationalités à Vienne, symbolisée par l'arrivée du troublant Mandryka, de ses lointaines contrées slaves, dans la vie d'une insensible jeune Viennoise. Dans L'Homme difficile également, Vienne est muséifiée en ville de l'aristocratie, envers laquelle Hofmannsthal est à la fois solidaire et critique. 
L'attachement se mue en satire de ses vaines aspirations, manifestée dans l'usage comique d'une langue désuète, hybride entre l'allemand et le français. Le choix d'évoquer le traumatisme de la guerre et de dépeindre l'aristocratie engluée dans un monde dont elle refuse de voir l'effondrement imminent (l'action se déroule en 1917) marque la volonté de faire de cette Vienne scénique un lieu représentatif de la crise du monde contemporain.

\section{Vienne et ses miroirs}

Il parait essentiel, face à cette mosaïque, de s'interroger sur les villes désignées comme les comparants de cette Vienne qui se proclame moderne mais qui chez Hofmannsthal revendique surtout ses traditions, et qui rétrospectivement a parfois pâti d'une image de frilosité face aux avant-gardes contemporaines. Le regard de Hofmannsthal sur les différentes capitales culturelles obéit à une stratégie de différenciation, nécessaire pour penser l'identité viennoise éclatée. Si l'on reproche souvent à la Vienne du Tournant du siècle son conservatisme provincialiste, Hofmannsthal refuse cette tentation (RAIII, p. 184) et, conscient que l'art moderne n'est paradoxalement pas tout à fait entré dans sa ville (RAI, p. 533), présente au contraire Vienne en prototype de la métropole, à la population laborieuse plongée dans la solitude, dont il diagnostique la répulsion pour les formes artistiques et politiques langagières (RAI, p. 479). L'Autriche, pays romantique (RAII, p. 35), s'oppose à l'Allemagne du Nord. En revanche, Hofmannsthal, qui insiste sur l'absurdité à assimiler l'Autriche tout entière à sa capitale (RAII, p. 391), révèle un "climat de l'âme » commun entre Vienne, d'autres villes autrichiennes (RAII, p. 266 ; RAIII, p. 178), dont Salzbourg, « cœur du cœur de l'Europe », dont il a fondé le festival pour compenser l'hégémonie de celui de Bayreuth, en réponse aux besoins d'un public hétérogène (RAII, p. 255 ; p. 258), et les villes d'Allemagne du Sud, Munich, Stuttgart (RAIII, p.180). Cette variété est fondatrice dans son désir de créer une nouvelle scène théâtrale germanophone, car sa tradition théâtrale humaniste séculaire est, au prix de la constitution d'un répertoire spécifique, capable de rivaliser avec la scène parisienne (RAII, p. 240). Berlin, «ville des parvenus » (RAI, p. 103), spécialisée dans la représentation réaliste du pathologique et du bizarre (RAII, p. 231), si elle a joué un rôle crucial pour le théâtre hofmannsthalien dans ses collaborations avec le Viennois Reinhardt exilé dans la capitale allemande, n'est nullement érigée en modèle pour la scène viennoise en déclin. C'est d'ailleurs New York qui obtient le statut de capitale du monde (RAII, p. 484).

Mais c'est Paris, ville de la sociabilité traversée par de grandes solitudes (RAII, p. 185), ville du traditionalisme au même titre que Vienne, qui bénéficie d'un statut de référence. Objets d'une reconstitution historique ludique de la cour de Louis XIV dans le «faux Molière » de Hofmannsthal, Die Lästigen (Les Fâcheux), la scène parisienne, le Louvre et Versailles (RAII, p. 327 ; p. 339) font figure à la fois de modèles et de rivaux. Paris est louée pour son architecture et sa géographie haussmaniennes, aux lignes simples mais grandioses qui mettent en valeur l'unité de la ville, édifiée de règnes en règnes, ce qui tranche avec la profusion architecturale du centre de Vienne (RAII, p. 387). Dans son combat pour tenir son rang après guerre, Vienne, « capitale artistique et spirituelle du Sud de l'Europe ", " centre allemand de la vie théâtrale depuis la fin du XVIII ${ }^{\mathrm{e}}$ siècle ", telle une "créature menacée qui a recours à ses dernières ressources ", 
prend acte de ce que sa seule rivale est Paris, la seule scène à se tourner encore vers toute la communauté des spectateurs (RAII, p. 279).

\section{Vienne la morte?}

Existe-t-il une identité viennoise à l'heure moderne pour Hofmannsthal, qui refuse de succomber aux leurres de la Modernité ? Malgré son attachement à l'Empire, il est conscient de la scission viennoise démontrée par Claudio Magris ${ }^{16}$ entre une facticité, une séduisante superficialité de sa vie culturelle, et une crise socio-politique et éthicoesthétique, dans un pessimisme qui jamais cependant ne sombre dans le nihilisme, mais promeut un classicisme moderne. Derrière la louange ambivalente d'une Vienne conçue comme une construction esthétique, baroque, entre l'Orient et l'Occident, fascinante en raison même de sa décadence, on perçoit la prise de conscience des enjeux des nationalités, qui jette sur les villes de l'Est un regard inquiet mais fasciné. La vision apocalyptique se surimpose à une vision utopique, où Vienne, non tant en réaction contre la montée de Berlin, que dans un désir de rapprochement avec la latinité au sein de la germanité, se constitue une identité dans une heureuse communion avec les villes d'Allemagne du Sud, et dans une relation d'admiration et de saine rivalité avec Paris.

\section{NOTES}

1. Karl Kraus, La Littérature démolie, traduit par Yves Kobry, Paris, Rivages poche, 1993.

2. Reden und Aufsätze II, Gesammelte Werke in zehn Einzelbänden, Frankfurt a. M., Fischer, 1979, p. 347. Les trois volumes de Reden und Aufsätze sont notés RAI, RAII, RAIII. Sauf mention contraire, c'est nous qui traduisons.

3. Werner Volke, Hugo von Hofmannsthal, traduit par Jean-Yves Masson, Nîmes, Jacqueline Chambon, 1996.

4. Henri Meschonnic, Modernité modernité, Paris, Verdier, 1988 ; Gallimard, « Folio Essais », 1994, p. 174.

5. Hermann Broch, « Hofmannsthal et son temps ", Création littéraire et connaissance, traduction d'Albert Koch, Paris, Gallimard, « Tel », 1966.

6. Buch der Freunde, RAIII, p. 279-280.

7. «Von einem kleinen Wiener Buch», RAI, p. 161.

8. « Wiener Brief II », RAII, p. 195-196.

9. «Internationale Kunst-Ausstellung 1894 », RAI, p. 542-544.

10. Ibid., p. 542-543.

11. Erzählungen. Erfundene Gespräche und Briefe. Reisen, Gesammelte Werke, op. cit., p. 447.

12. «Philosophie des Metaphorischen », RAI, p. 192-193.

13. Cité par Jacques Le Rider, Modernité viennoise et crises de l'identité, Paris, PUF, "Quadrige », 2000 , p. 23.

14. «Eduard von Bauernfelds dramatischer Nachlass », RAI, p. 189.

15. Aufzeichnungen aus dem Nachlass, RAIII, p. 383. 
16. Claudio Magris, Le Mythe et l'Empire dans la littérature autrichienne moderne, traduit par Jean et Marie-Noëlle Pastoureau, Paris, «L'Arpenteur », Gallimard, 1991.

\section{RÉSUMÉS}

Hofmannsthal, Viennois de naissance, n'a pas écrit de texte majeur sur sa ville natale, à laquelle il accorde pourtant une attention jamais démentie. La crise du début du $x^{\mathrm{e}}$ siècle le conduit à chercher ce qui fait la spécificité de l'histoire, de la langue, de la psychologie, des mœurs de sa ville : il évoque souvent la vie intellectuelle et artistique de Vienne, sa peinture, sa musique, sa littérature, son architecture. Représentant d'une « Modernité viennoise » dont il ne se fait jamais le porte-parole, s'il jette sur le passé grandiose de Vienne un regard admiratif, il se montre critique envers son actualité culturelle, et conscient des fragiles fondements de l'équilibre de la Double Monarchie: la mythification nostalgique et utopique de Vienne en ville baroque, orientalisante, aristocratique, séductrice, poétique, puis en mosaïque des peuples, coexiste avec une démystification scénique de sa vie intellectuelle en déclin, de son vain attachement à un passé glorieux. Le fantasme de la "ville morte" semble pourtant se résorber dans la revendication des traditions théâtrales communes entre Vienne, les villes d'Autriche et d'Allemagne du Sud, non tant en opposition au Berlin des avant-gardes que dans une fascination pour la latinité de Venise, et surtout de Paris, auréolée de ses scènes théâtrales.

Der in Wien geborene Hofmannsthal hat trotz einem nie nachlassenden Interesse für seine Vaterstadt keinen großen Text darüber geschrieben. Die Krise am Anfang des XX. Jahrhunderts führt ihn zur Untersuchung der Spezifik der Geschichte, Sprache, Psychologie und Sitten Wiens: oft erwähnt er das intellektuelle und künstlerische Leben in Wien, die Malerei, die Musik, die Literatur, die Architektur. Als Vertreter der Wiener Moderne, aber nie als ihr Wortführer, zeigt er sich trotz seiner Bewunderung der grandiosen Vergangenheit Wiens kritisch gegenüber der Wiener Gegenwartskunst und ist sich der zerbrechlichen Basis der kaiserlichen und königlichen Stabilität bewusst: Die sehnsüchtige und utopische Mythisierung Wiens als einer barocken, orientalisierenden, aristokratischen, verführerischen, poetischen Stadt und dann als einer Vielvölkerstadt steht neben einer szenischen Entmystifizierung des verfallenden intellektuellen Lebens, der eitlen Zuneigung zur ruhmvollen Vergangenheit. Die Wahnvorstellung von der «toten Stadt» scheint trotzdem in der Forderung gemeinsamer theatralischer Traditionen zwischen Wien und den Städten Österreichs und Süddeutschlands überwunden, weniger als Gegenentwurf zum avantgardistischen Berlin, als aus einer Faszination für die Latinität Venedigs und des von seinen Theaterbühnen gekrönten Paris.

\section{INDEX}

Mots-clés : Vienne 
AUTEURS

AUDREY GIBOUX

Université de Paris IV 